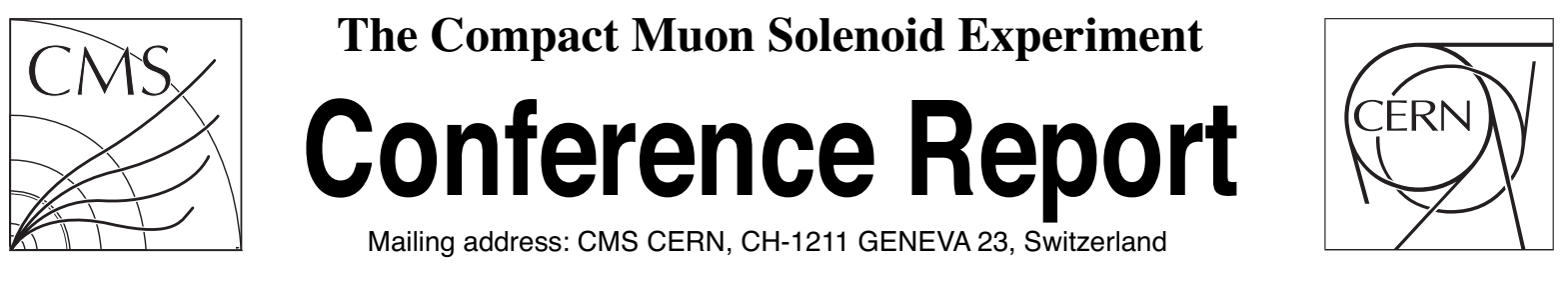

23 December 2019 (v4, 08 April 2020)

\title{
Precision Timing with LYSO Ce Crystals and SiPM Sensors in the CMS MTD Barrel Timing Layer
}

Martina Malberti for the CMS Collaboration

\begin{abstract}
The Compact Muon Solenoid (CMS) detector at the CERN Large Hadron Collider (LHC) is undergoing an extensive Phase II upgrade program to prepare for the challenging conditions of the HighLuminosity LHC (HL-LHC). In particular, a new timing detector will measure minimum ionizing particles (MIPs) with a time resolution of $30-40 \mathrm{ps}$ and hermetic coverage up to a pseudo-rapidity of I?I=3. The precision time information from this MIP Timing Detector (MTD) will reduce the effects of the high levels of pile-up expected at the HL-LHC and will bring new and unique capabilities to the CMS detector. The central Barrel Timing Layer (BTL) will be based on LYSO Ce crystals read out with silicon photomultipliers (SiPMs). The BTL will use elongated crystal bars, with double-sided read out, with a SiPM on each end of the crystal, in order to maximize detector performance within the constraints of space, cost, and channel count. This unusual geometry enables the instrumentation of large surfaces while minimizing the active area of the photodetectors, and thus noise and power consumption. We will present an overview of the MTD BTL design and will detail the extensive R and D studies carried out to optimize the MTD BTL crystal-based technology and test beam results in which the goal of $30 \mathrm{ps}$ timing resolution has been achieved. We will also present progress in the development of the dedicated readout electronics for the BTL.
\end{abstract}




\section{Precision timing with LYSO:Ce crystals and SiPM sensors in the CMS MTD barrel timing layer}

To cite this article: M. Malberti 2020 JINST 15 C04014

View the article online for updates and enhancements.

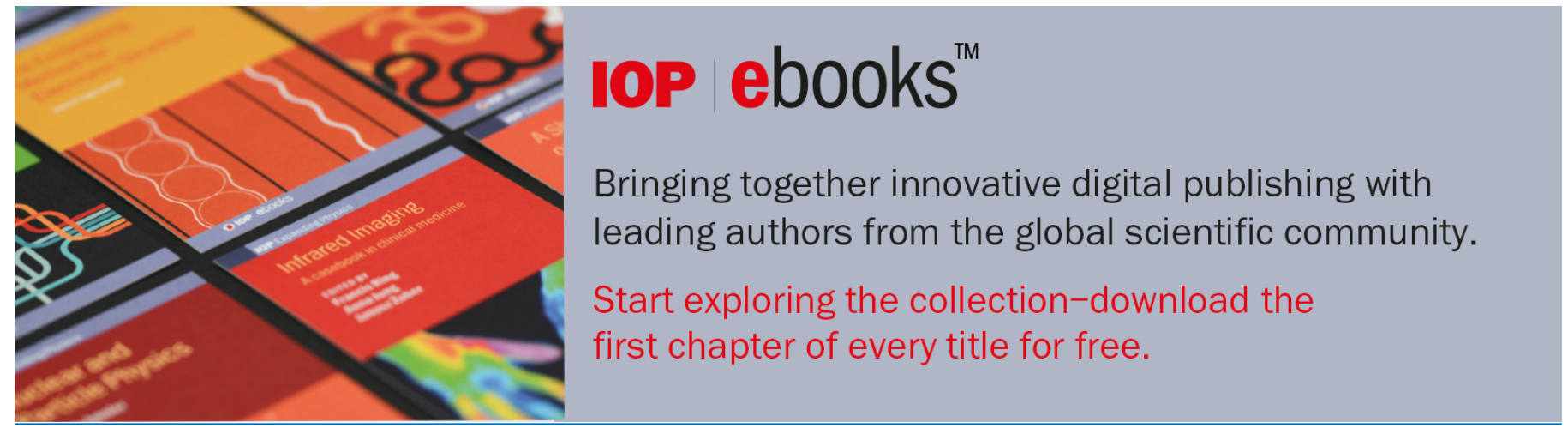

This content was downloaded from IP address 95.246 .143 .1 on $08 / 04 / 2020$ at 13:00 


\title{
Precision timing with LYSO:Ce crystals and SiPM sensors in the CMS MTD barrel timing layer
}

\author{
M. Malberti on behalf of the CMS collaboration \\ Istituto Nazionale di Fisica Nucleare, Sezione di Milano Bicocca, \\ Piazza della Scienza 3, 20126 Milano, Italy \\ E-mail: martina.malberti@mib.infn.it
}

\begin{abstract}
Aвstract: The Compact Muon Solenoid (CMS) detector at the CERN Large Hadron Collider (LHC) is undergoing an extensive Phase II upgrade program to prepare for the challenging conditions of the High-Luminosity LHC (HL-LHC). In particular, a new timing detector will measure minimum ionizing particles (MIPs) with a time resolution of $\sim 30-40 \mathrm{ps}$ and hermetic coverage up to a pseudorapidity of $|\eta|=3$. The precision time information from this MIP Timing Detector (MTD) will reduce the effects of the high levels of pileup expected at the HL-LHC and will bring new and unique capabilities to the CMS detector. The central Barrel Timing Layer (BTL) will be based on LYSO:Ce crystals read out with silicon photomultipliers (SiPMs). The BTL will use elongated crystal bars, with double-sided read-out, with a SiPM on each end of the crystal, in order to maximize detector performance within the constraints of space, cost, and channel count. This unusual geometry enables the instrumentation of large surfaces while minimizing the active area of the photodetectors, and thus noise and power consumption. We will present an overview of the MTD BTL design and will detail the extensive R\&D studies carried out to optimize the MTD BTL crystal-based technology and test beam results in which the goal of 30 ps timing resolution has been achieved.
\end{abstract}

KeYwords: Photon detectors for UV, visible and IR photons (solid-state); Scintillators, scintillation and light emission processes (solid, gas and liquid scintillators); Timing detectors 


\section{Contents}

1 Motivations for precision timing at the HL-LHC 1

2 Overview of the CMS MIP timing detector 3

3 The MTD barrel timing layer 3

3.1 BTL sensors and layout 3

3.2 BTL performance in test beams 5

3.3 BTL performance evolution 5

4 Conclusions $\quad 7$

\section{Motivations for precision timing at the HL-LHC}

The CERN Large Hadron Collider (LHC) is undergoing a significant upgrade of the machine and injectors to increase the beam intensity. The High-Luminosity LHC (HL-LHC) phase [1] is expected to start in 2027, it will bring the peak instantaneous luminosity up to $7.5 \times 10^{34} \mathrm{~cm}^{-2} \mathrm{~s}^{-1}$ and will provide an integrated luminosity of $3000 \mathrm{fb}^{-1}$ in 10 years of operation. The increase in instantaneous luminosity will come along with an increase in the number of simultaneous interactions per bunch crossing (known as pileup) from the current 40 up to 200, implying a vertex density five times higher than the one at the current LHC. The probability density functions of the line density along the beam axis for the pileup of the LHC and for pileup 140 and 200 are shown in figure 1.

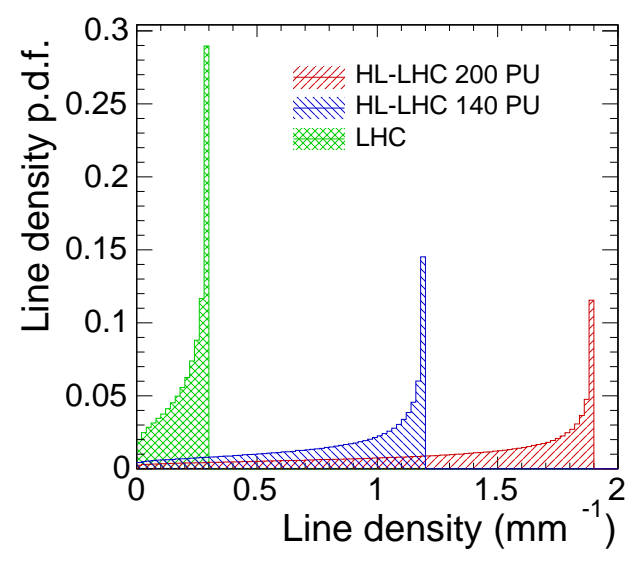

Figure 1. Probability density functions of the vertex density along the beam axis for the pileup at the LHC and for the pileup of 140 and 200 at the HL-LHC [2]. 
With such levels of vertex densities $\left(>1 \mathrm{~mm}^{-1}\right)$ a significant degradation in most event observables is expected because a large number of pileup tracks will be incorrectly associated to the primary vertex of interest. A powerful tool to mitigate the effects of pileup is the time tagging of tracks with a resolution of few tens of picoseconds. Due to the longitudinal spread of the interactions along the beam direction, interactions are spread in time with an RMS of about 200 ps. Precision timing would allow to resolve vertices that are close in space but separated in time and to suppress spurious pileup tracks exploiting the compatibility between the time of tracks and of vertices, as illustrated in figure 2 .

To address the pileup challenge, the CMS [3] collaboration is thus planning to add a dedicated timing layer that will measure minimum ionizing particles (MIPs) with a time resolution of 30-40 ps, referred in the following as the MIP Timing Detector (MTD) [2].
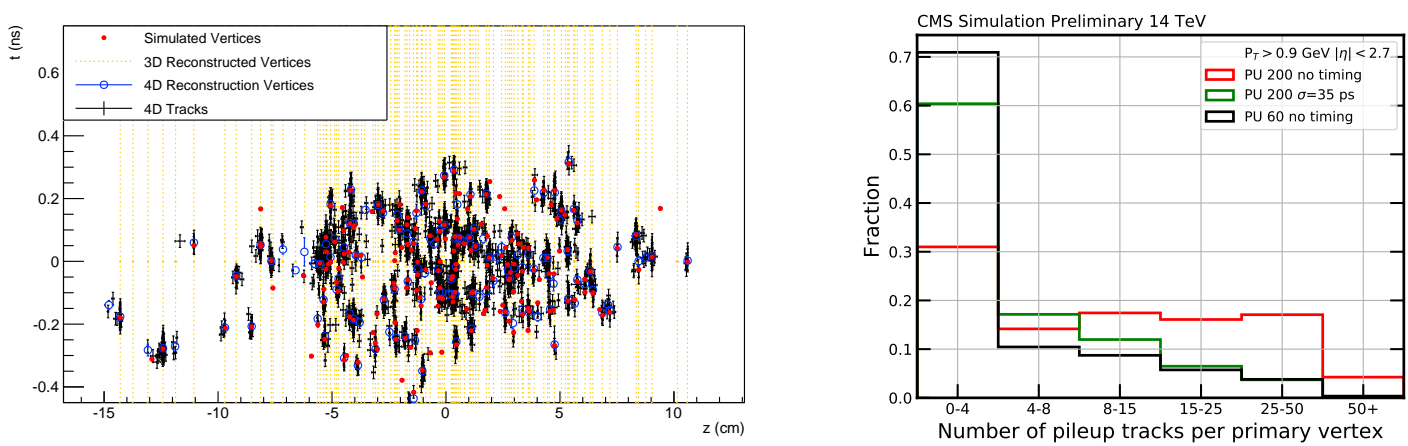

Figure 2. Left: simulated and reconstructed vertices in a 200 pileup event assuming a MIP timing detector covering the barrel and endcaps. The vertical yellow lines indicate 3D-reconstructed vertices, with instances of vertex merging visible throughout the event display. Right: distribution of the number of incorrectly associated tracks with the use of a $3 \sigma$ (where $\sigma=35 \mathrm{ps}$ ) selection on timing information and without use of timing information. The vertical axis is the fraction of primary vertices which have the number of pileup tracks shown on the horizontal axis associated to them [2].

Precision timing will bring many benefits to the full CMS physics program. The removal of pileup tracks inconsistent with the hard interaction improves the quality of the event reconstruction providing a higher b-tagging efficiency, improved identification and isolation of leptons and photons, better rejection of fake jets due to pileup, improved missing transverse momentum resolution. All these improvements on physics objects are expected to bring a gain between $10 \%$ and $20 \%$ in the statistical sensitivity for many Higgs boson measurements, corresponding to an increase of about $26 \%$ in the effective luminosity [2]. In addition, the usage of timing will enable also velocity measurement for low transverse momentum hadrons (pions, kaons, or protons), which is beneficial for the Heavy Ion physics and for specialized QCD studies in pp collisions. The 4D vertex reconstruction of primary and secondary vertices will also provide a unique tool to extend the potential of searches for long-lived particles (LLPs) predicted by several BSM physics models thanks to the capability to reconstruct, under certain assumptions, the LLP's mass. 


\section{Overview of the CMS MIP timing detector}

The CMS MIP timing detector consists of a thin layer placed between the tracker and the calorimeters. It is designed to efficienctly measure minimum ionizing particles with a time resolution of $30-40 \mathrm{ps}$ and with a hermetic coverage up to $|\eta|=3$. The MTD is divided in two parts, the barrel part (up to $|\eta|=1.45$ ) based on scintillating crystals coupled to silicon photomultipliers (SiPMs) and the endcap, covering the region between $|\eta|=1.6$ and $|\eta|=3$, based on low gain avalanche photodiodes. A schematic view of the MTD detector is shown in figure 3. Different detector technologies are chosen to cope with the different radiation levels and with additional constraints from integration within the existing CMS detector, cost, and power budgets.

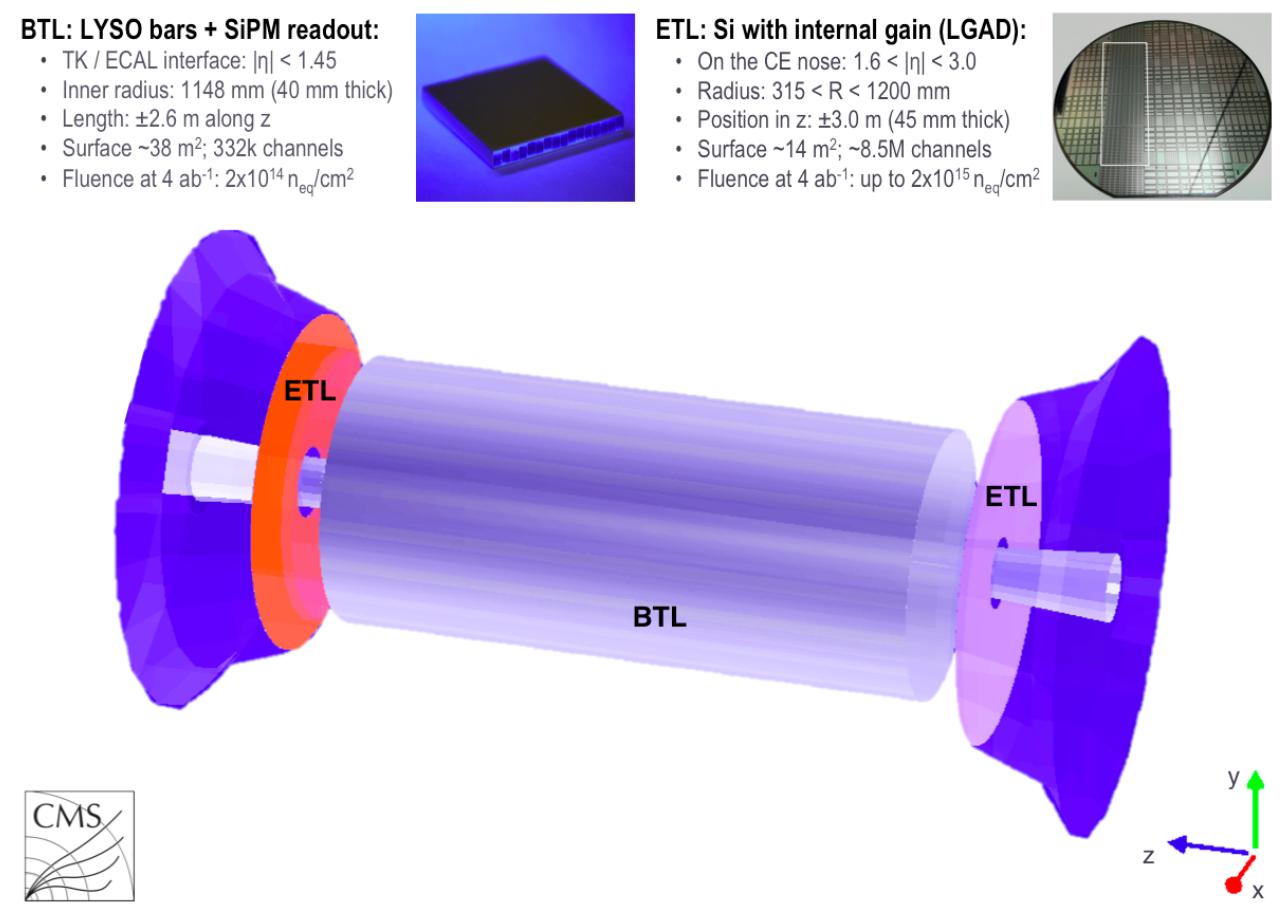

Figure 3. A schematic view of the MTD detector comprising a barrel timing layer (grey cylinder), at the interface between the tracker (inside) and the electromagnetic calorimeter (outside), and two silicon endcap timing layers (orange and light violet disks) in front of the endcap calorimeter [2].

\section{The MTD barrel timing layer}

\subsection{BTL sensors and layout}

The barrel timing layer (BTL) consists of a cylindrical detector of $5200 \mathrm{~mm}$ length and $1148 \mathrm{~mm}$ radius, which will be mounted on the inner surface of the tracker support tube, as shown in figure 4 . It will be a single layer, $40 \mathrm{~mm}$ thick, segmented in 72 trays. Each tray consists of six Read-out Units with 24 modules each. A sensor module is an array of 16 crystals.

The basic active elements of the BTL are LYSO:Ce crystal bars of $57 \mathrm{~mm}$ length, $3.12 \mathrm{~mm}$ width and $3 \mathrm{~mm}$ average thickness (figure 5-left), wrapped with reflective material and read out at both ends 

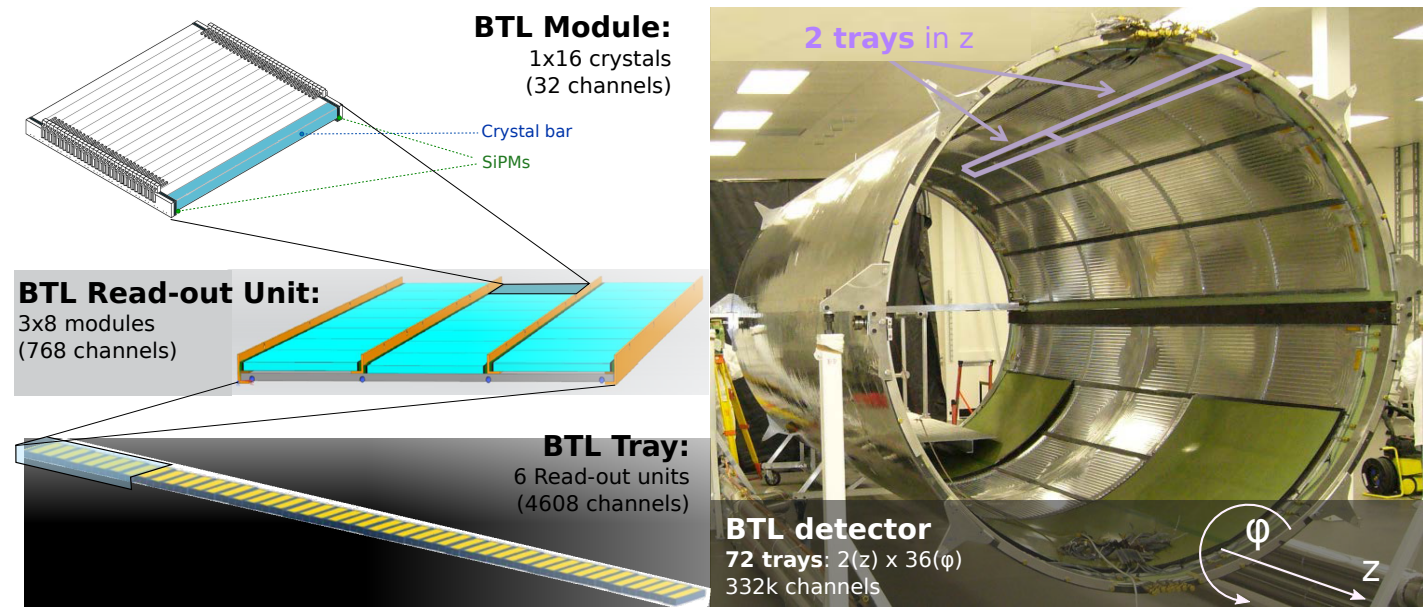

Figure 4. Overview of the BTL showing (left) the hierarchical arrangement of the various components, bars, modules, and Read-out Units, and (right) trays (purple rectangles near the top), inside the tracker support tube. [2].

by SiPMs. LYSO:Ce was chosen for its excellent radiation tolerance (figure 5-right), high density $\left(>7.1 \mathrm{~g} / \mathrm{cm}^{3}\right)$, high light yield $(\sim 40000$ photons $/ \mathrm{MeV})$ and fast rise time $(\sim 100 \mathrm{ps})$ and relatively fast decay time (40 ns), along with cost and mass production capability considerations. The thickness of the crystals along the $z$ axis of the CMS detector is optimized to maintain an approximately constant slant depth crossed by particles and to limit the amount of material in front of the CMS electromagnetic calorimeter so as to have a negligible impact on its energy resolution. The different thicknesses are $3.7 \mathrm{~mm}$ for $|\eta|<0.7,3.0 \mathrm{~mm}$ for $0.7 \leq|\eta| \leq 1.1$ and $2.4 \mathrm{~mm}$ for $|\eta|>1.1$.
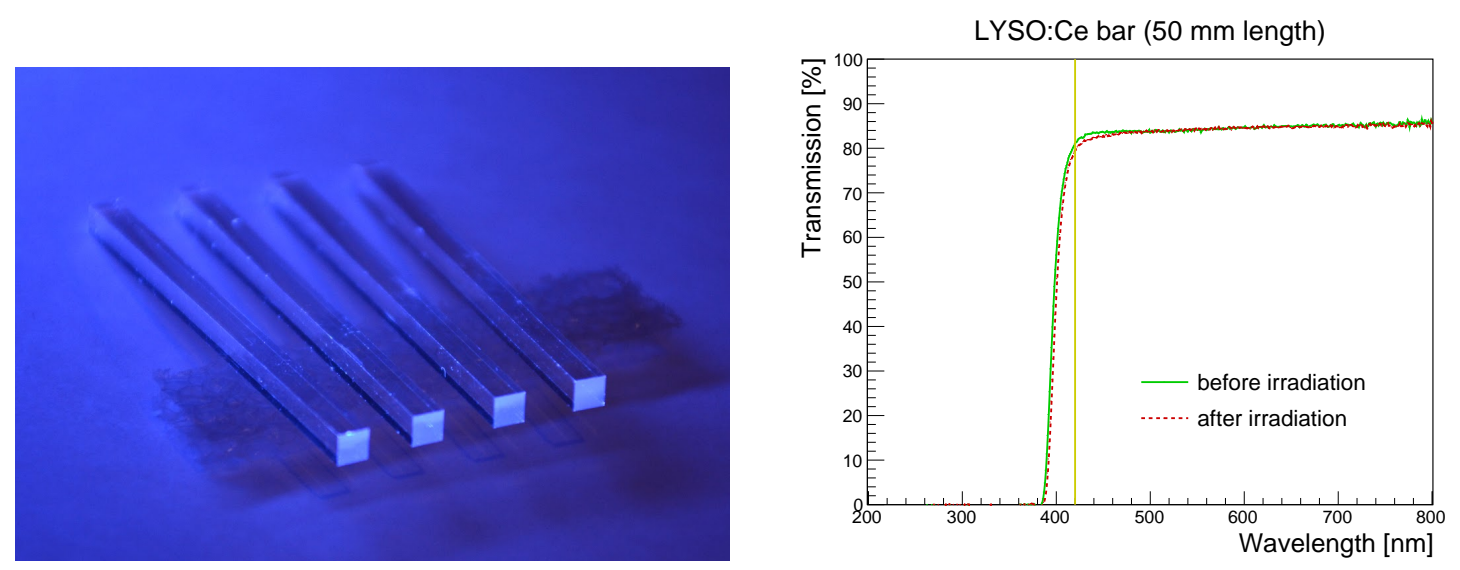

Figure 5. Left: picture of four LYSO:Ce crystal bars without wrapping under UV illumination. Right: transparency measured across a $50 \mathrm{~mm}$ long LYSO:Ce crystal bar, before and after irradiation with $24 \mathrm{GeV}$ protons to a fluence of $2 \times 10^{13} \mathrm{~cm}^{-2}$ [2].

Silicon photomultipliers are compact, fast, and insensitive to magnetic fields. They have been proven capable to withstand the integrated radiation levels of about $2 \times 10^{14} 1 \mathrm{MeV}$ neq $/ \mathrm{cm}^{2}$ foreseen for the BTL at the end of the detector operation. The optimal SiPM cell size that maximizes the 
photon detection efficiency, ensures high radiation tolerance against the dark count induced noise, and provides a high dynamic range, was found to be $15 \mu \mathrm{m}$. A total number of $331776 \mathrm{SiPMs}$ of about $3 \times 3 \mathrm{~mm}^{2}$ area is forseen. Different SiPM technologies are under consideration for BTL, including the NUV-HD (thin-epi) SiPM from Fondazione Bruno Kessler (FBK) and the S12572 and HDR2 from Hamamatsu Photonics (HPK).

The choice of a high aspect ratio geometry for the BTL sensors allows to exploit the total internal reflection to enhance the light collection and to minimize the ratio between the SiPM area over the crystal area to cope with power consumption constraints and yielding to overall better timing performance after irradiation.

The SiPMs are read out by a dedicated chip called TOFHIR (i.e. Time-Of-Flight at High Rates), based on the TOFPET2 board [4] used in LYSO TOFPET and adapted to manage the higher rates and radiation conditions expected at the HL-LHC. Each TOFHIR provides amplitude and time measurement for 32 channels. The time measurement is based on discrimination of the leading edges (LE) of the pulses, followed by a time-to-digital converter (TDC) with 20 ps binning. A dedicated noise filtering is implemented in the ASIC, with an architecture similar to the first stage of a constant fraction discriminator, to reduce the baseline fluctuations due to the SiPM dark counts.

\subsection{BTL performance in test beams}

Extensive test beam campaigns have been conducted to prove the target resolution of about $30 \mathrm{ps}$. Figure 6 shows the time stamp of one SiPM in a bar as a function of the impact point position of the particle along the bar. The time stamp measured at a single end depends on the distance between the point where the scintillation photons are emitted and the SiPM, due to the propagation time of optical photons within the crystal. The average of the times measured at the two ends of a bar provides a uniform time response, as shown in figure 6 , and the optimal time resolution $(<30 \mathrm{ps})$ across the full bar lenght. The combination of the two SiPM measurements improves the overall time resolution by $\sqrt{2}$ with respect to the single SiPM, since the dominant stochastic fluctuations from photo-statistics and noise are uncorrelated for the two SiPMs. In addition, since the time difference between the two time stamps is strongly correlated with the impact point position along the bar, the sensors will provide tracking information with a precision better than $5 \mathrm{~mm}$.

\subsection{BTL performance evolution}

The time resolution per track is given by the sum in quadrature of different terms:

$$
\sigma_{\mathrm{t}}^{\mathrm{BTL}}=\sigma_{\mathrm{t}}^{\text {clock }} \oplus \sigma_{\mathrm{t}}^{\text {digi }} \oplus \sigma_{\mathrm{t}}^{\text {ele }} \oplus \sigma_{\mathrm{t}}^{\text {phot }} \oplus \sigma_{\mathrm{t}}^{\mathrm{DCR}}
$$

The contributions from clock digitization (15 ps), digitization ( $7 \mathrm{ps})$ and electronics ( $8 \mathrm{ps}$ ) are small and are all expected to be constant across the detector lifetime. The driving terms in time resolution are the photo-statistic term and the noise term due to SiPM dark counts. The photostatistics term amounts to about 25-30 ps. The noise term is negligible at startup and increases with increasing radiation damage becoming the dominant contribution at the end of operations (about $50 \mathrm{ps}$ after $3000 \mathrm{fb}^{-1}$ ). The relative contribution of all these terms to the total time resolution is shown in figure 7 as function of the integrated luminosity. 

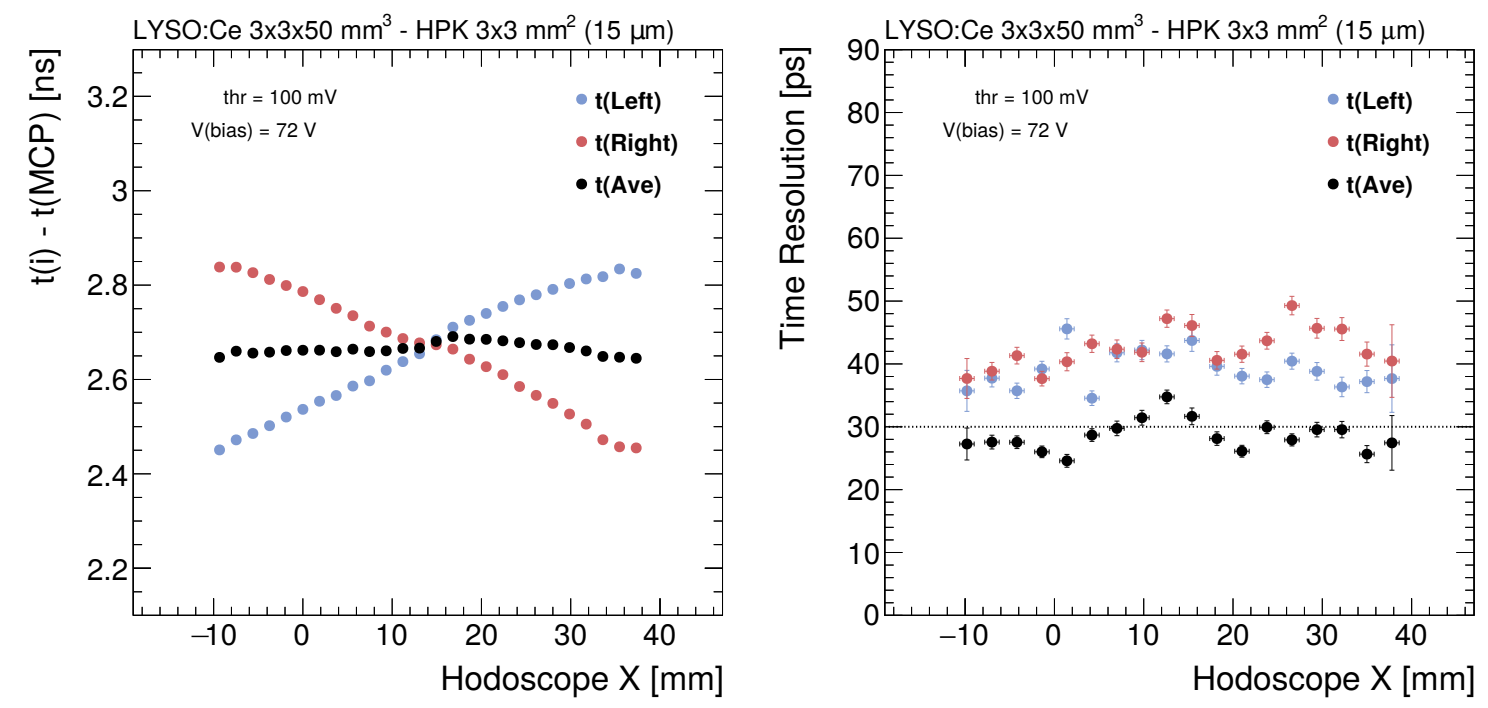

Figure 6. Time stamp (left) and time resolution (right) from the left and right SiPMs in one bar and from the average time stamp, as a function of the impact point $\mathrm{X}$ along the crystal bar axis [2].

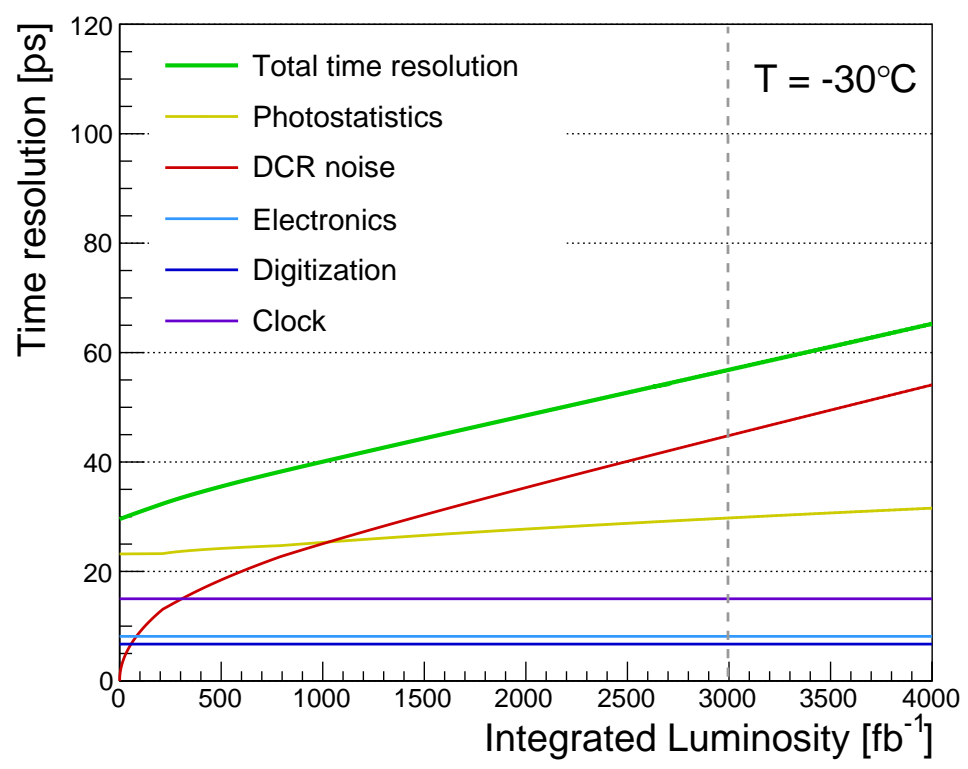

Figure 7. Evolution of different terms contributing to the BTL time resolution as a function of integrated luminosity. The two time measurements from the SiPMs at the opposite ends of a LYSO:Ce crystal bar are combined in a single measurement. The curves are calculated for the SiPM type HDR2-015 from Hamamatsu [2]. 
Different approaches are foreseen in order to mitigate the DCR noise:

- Cooling. Since the DCR increases by roughly a factor of two every $7-10^{\circ} \mathrm{C}$, the detector will be operated at temperature of about $-30^{\circ} \mathrm{C}$.

- SiPM annealing. Periods of annealing at room temperature are foreseen during shutdowns.

- Adjustment of the SiPMs operating voltage. Whereas the DCR increases with the fluence, the SiPM operating voltage will have to be decreased by about $2 \mathrm{~V}$ throughout the detector lifetime in order to maintain the DCR level within a range manageable by the ASIC as well as to limit the power dissipated by the SiPM through heat within the design specification.

- Noise filtering in the ASIC. The circuit is expected to reduce the high frequency dark count noise by a factor of the order of two.

As a result, the time resolution of the BTL is expected to vary between 30 and 60 ps across the entire period of operation during the HL-LHC.

\section{Conclusions}

The CMS upgrade for HL-LHC will include a new MIP timing detector to measure the time of arrival of charged particles with a target resolution of about $30 \mathrm{ps}$ and a hermetic coverage. The full CMS physics program will largely benefit from this new detector that will bring improved pileup mitigation and physics object reconstruction performances, with a corresponding increase in effective luminosity, and will enable particle identification with time-of-flight capabilities and new searches for long lived particles. The central part of the MTD will be based on LYSO:Ce crystal bars with double-end SiPM read-out. Successfull test beam campaigns have been conducted in the past years demonstrating that the target time resolution is achievable. While, the LYSO crystals and SiPM qualification is ongoing, more complete full-system tests are planned for 2020. According to the current schedule, the BTL construction will start in 2021.

\section{References}

[1] G. Apollinari, O. Brüning, T. Nakamoto and L. Rossi, High Luminosity Large Hadron Collider HL-LHC, CERN yellow report CERN-2015-005, CERN, Geneva, Switzerland (2015) [arXiv: 1705.08830].

[2] CMS collaboration, A MIP timing detector for the CMS phase-2 upgrade, CERN-LHCC-2019-003, CERN, Geneva, Switzerland (2019).

[3] CMS collaboration, The CMS experiment at the CERN LHC, 2008 JINST 3 S08004.

[4] M.D. Rolo et al., TOFPET ASIC for PET applications, 2013 JINST 8 C02050. 